\title{
Lentivirus-mediated RNA silencing of c-Met markedly suppresses peritoneal dissemination of gastric cancer in vitro and in vivo
}

\author{
Xiao-lei WANG ${ }^{1, *}$, Xi-mei CHEN ${ }^{1}$, Jian-ping FANG ${ }^{2}$, Chang-qin YANG ${ }^{1}$ \\ ${ }^{1}$ Department of Gastroenterology, Institute of Digestive Disease, Tongji Hospital affiliated to Tongji University, Shanghai 200065, China; \\ ${ }^{2}$ Department of Pathology, Tongji Hospital affiliated to Tongji University, Shanghai 200065, China
}

\begin{abstract}
Aim: To investigate the expression of c-Met in peritoneal free cancer cells isolated from human gastric cancer ascites, and its relationship to peritoneal dissemination of gastric cancer.

Methods: Peritoneal free cancer cells (PFCCs) were isolated from ascites specimens of gastric cancer patients. c-Met expression in PFCCs was detected with immunocytochemistry. In human gastric cancer cell line SGC7901, c-Met expression was detected using RT-PCR and Western blot, and was suppressed with lentivirus-mediated RNAi. The proliferation of SGC7901 cells was measured using MTT assay, and the invasion ability was detected with invasion assay. The adhesion of SGC7901 cells to peritoneum was observed in human peritoneal mesothelial cells (HPMCs) monolayer in vitro and in mice in vivo.

Results: PFCCs were isolated from ascites of 6 out of 10 gastric cancer patients. c-Met expression in PFCCs was detected in 5 of the 6 gastric cancer patients. In SGC7901 cells, Lentivirus-mediated RNAi significantly reduced both c-Met mRNA and protein expression, which resulted in suppressing the cell proliferation, invasion and adhesion to peritoneum. The expression of $\alpha 3 \beta 1$ integrin and E-cadherin was significantly inhibited in SGC7901 cells transfected with Lenti-miRNAc-Met. In the peritoneal dissemination model of gastric cancer, intraperitoneal injection of Lenti-miRNAc-Met markedly suppressed the tumor progression of SGC7901 cells.

Conclusion: c-Met is expressed in PFCCs from the ascites of gastric cancer patients. Down-regulation of c-Met expression markedly suppresses the multistep process of peritoneal dissemination, thus may be a potential target for the treatment of gastric cancer.
\end{abstract}

Keywords: gastric cancer; peritoneal free cancer cell; ascites; peritoneal dissemination; c-Met; lentivirus; RNA interference

Acta Pharmacologica Sinica (2012) 33: 513-522; doi: 10.1038/aps.2011.205; published online 12 Mar 2012

\section{Introduction}

Peritoneal metastasis frequently occurs in patients with gastric cancer and is associated with a poor prognosis. Despite advances in chemotherapy, including neoadjuvant intraperitoneal systemic chemotherapy for peritoneal dissemination of gastrointestinal cancers, the results are unsatisfactory, and a standard and effective treatment has not been developed.

The establishment of peritoneal dissemination is a multistep process that includes the detachment of cancer cells from primary cancer, the adhesion and interaction of peritoneal free cancer cells (PFCCs) and mesothelial cells on the peritoneal surface, the penetration of free cancer cells into the submesothelial space, the attachment of cancer cells to the exposed submesothelial basement membrane, and the invasion of

\footnotetext{
* To whom correspondence should be addressed.

E-mail xlwang2006@163.com

Received 2011-09-28 Accepted 2011-12-19
}

the peritoneal-blood barrier finally ${ }^{[1]}$. Many molecules are involved in this process. For example, E-cadherin is the key molecule for detachment ${ }^{[2]}$. CD44 is considered important for the adhesion and interaction between cancer cells and mesothelial cells ${ }^{[3]}$. Integrins, a family of adhesion receptors consisting of 18 a and $8 \beta$ subunits that form 24 distinct integrins, mediate cell-cell and cell-extracellular matrix (ECM) connections and are involved in the penetration of cancer cells into the submesothelial basement membrane ${ }^{[4,5]}$.

Recently, c-Met, a receptor tyrosine kinase, and its ligand, hepatocyte growth factor (HGF), have become leading candidates for molecular targeted cancer therapies ${ }^{[6]}$ with the potential to block peritoneal metastasis. c-Met and HGF participate in most stages of malignant progression, including adhesion, degradation of ECM, promotion of cell mobility and the interaction between cancer cells and peritoneal mesothelial cells. Thus, integrins are involved with adhesion, invasion, dissemination and metastasis ${ }^{[6]}$. Many human cancers, includ- 
ing ovarian cancer ${ }^{[7]}$, gastric cancer ${ }^{[8]}$, esophageal adenocarcinoma ${ }^{[9]}$, and colorectal cancer ${ }^{[0]}$, have c-Met overexpression in the primary cancer tissues, which is also associated with poor outcome. Moreover, an effective therapy which targeted c-Met in vivo demonstrated the ability to inhibit peritoneal dissemination and invasion in ovarian cancer xenografts ${ }^{[11]}$. The measurement of c-Met expression level in tissues is crucial to predict the treatment response to inhibitors. PFCCs are considered to play a key role in the process of peritoneal metastasis in gastric cancer, and the molecules expressed on the surface of PFCCs usually provide important clues for targeted therapy ${ }^{[12]}$. However, only approximately $10 \%$ of the malignant cells in the peritoneal cavity or ascites are detected by conventional methods. According to previous reports, immunomagnetic separation can efficiently improve the detection of rare free malignant cells in fluid and blood specimens ${ }^{[13,14]}$ and, thus, has the ability to study c-Met expression on PFCCs.

RNAi has been widely used as a powerful tool in gene function studies and as a potential treatment model for human cancers. miRNAs are members of a class of small regulatory RNAs and are targets of novel anticancer gene therapy by antisense molecules that can inhibit mRNA activity by mRNA cleavage or translational repression ${ }^{[15]}$. In this paper, we present a preliminary study on c-Met expression in PFCCs from gastric cancer patients and demonstrate a successful long-term efficient lentiviral miRNA (lenti-miRNA) system for silencing c-Met expression in the SGC7901 human gastric cancer cell line. We also evaluate c-Met as a therapeutic target in the treatment of gastric cancer peritoneal dissemination.

\section{Materials and methods}

Immunomagnetic isolation of PFCCs in ascites from gastric cancer patients

PFCCs from ascites of gastric cancer patients were isolated by the magnetic activated cell sorting (MACS) method. Briefly, ascites specimens were collected sterilely at the time of initial diagnosis, as confirmed by biopsy pathology, from 10 primary gastric cancer patients (Table 1). Samples and clinical

Table 1. PFCCs isolated by MACS and the c-Met/CK20 expression in the ascites of ten gastric cancer patients.

\begin{tabular}{cllll}
\hline $\begin{array}{c}\text { Patient } \\
\text { No }\end{array}$ & \multicolumn{1}{c}{ Histological type } & PFCCs & $\begin{array}{c}\text { c-Met } \\
\text { expression }\end{array}$ & $\begin{array}{c}\text { CK20 } \\
\text { expression }\end{array}$ \\
\hline 1 & Adenocarcinoma & Positive & Positive & Positive \\
2 & Signet-ring cell carcinoma & Negative & - & Negative \\
3 & Adenocarcinoma & Negative & - & Negative \\
4 & Adenocarcinoma & Negative & - & Negative \\
5 & Adenocarcinoma & Positive & Positive & Positive \\
6 & Signet-ring cell carcinoma & Negative & - & Negative \\
7 & Adenocarcinoma & Positive & Positive & Positive \\
8 & Signet-ring cell carcinoma & Positive & Negative & Positive \\
9 & Adenocarcinoma & Positive & Positive & Positive \\
10 & Adenocarcinoma & Positive & Positive & Positive \\
\hline
\end{tabular}

data were collected after informed consent was obtained. The ascites samples were centrifuged into pellets and resuspended in $10 \mathrm{~mL}$ of phosphate-buffered saline (PBS) supplemented with $0.5 \%$ bovine serum albumin (BSA). The red blood cells (RBCs) were lysed with fresh lysing buffer with a volume ratio of 1:5. The manufacturer's instructions (Miltenyi Biotec, Germany) for MACS were followed for cancer cell enrichment. Mononuclear cells (MNCs) were recovered by centrifugation $(200 \times g, 10 \mathrm{~min})$ through a Ficoll density gradient according to the manufacture's protocol. Next, $20 \mu \mathrm{L} \mathrm{FcR} \mathrm{blocking}$ reagent (Miltenyi Biotec) was added to block unspecific binding of MNCs to the microbeads. Anti-EpCAM conjugated with phycoerythrin (PE) monoclonal antibody (mAb) (Miltenyi Biotec) was incubated with the cells at a dilution of 1:10. Human peritoneal mesothelial cells (HPMCs) were labeled by anti-CD45 conjugated with fluorescein isothiocyanate (FITC) (Beijing CellChip Biotechnology Co, Ltd, China). The cells were incubated with $20 \mu \mathrm{L}$ anti-PE microbeads for $15 \mathrm{~min}$ on ice and then resuspended in $80 \mu \mathrm{L}$ MACS buffer. The magnetically labeled cells were washed with $1.5 \mathrm{~mL}$ MACS buffer and passed through a magnetic separation column (Miltenyi Biotec). The column was removed from the separator, and the positive fraction was eluted with the plunger supplied.

\section{Immunocytochemistry (ICC)}

Each isolated cell sample underwent routine hematoxylineosin (HE) staining to observe cell morphology under a light microscope. Expressions of CK20 and c-Met were evaluated by immunocytochemical staining using streptavidin-biotinperoxidase method in a cellular smear. First, cells were fixed in $4 \%$ paraformaldehyde. Next, primary anti-human CK20 (1:100) and c-Met (1:100) antibodies were incubated with the cells for $2 \mathrm{~h}$ at room temperature. The procedure was performed according to the manufacturer's instructions utilizing the horseradish peroxidase (HRP) immunohistochemistry (IHC) kit (Chemicon International Inc, Temecula, CA, USA). Cytoplasmic and/or cytomembrane immunostaining was considered positive. Negative control samples were processed similarly in the absence of primary antibody. The standard for assessment of malignancy was according to cytomorphology. The malignant cells were verified via light microscope criteria based on HE staining, including cellular pleomorphism, an altered nucleus and hyperchromasia.

\section{Cell line and cell culture}

The human gastric cancer cell line SGC7901 was obtained from Type Culture Collection of the Chinese Academy of Sciences (Institute of Biochemistry and Cell Biology, Shanghai, China). The tumor cells were cultured in RPMI-1640 media (PAA Laboratories $\mathrm{GmbH}$, Austria) supplemented with $10 \%$ fetal calf serum (FCS) and penicillin $(100 \mathrm{U} / \mathrm{mL}) /$ streptomycin $(100$ $\mu \mathrm{g} / \mathrm{mL})$ in an atmosphere of $37^{\circ} \mathrm{C}$ in $5 \% \mathrm{CO}_{2}$.

HPMCs were obtained following informed consent from the omental tissue of patients who underwent abdominal surgery for benign conditions. The HPMCs were isolated according to methods previously reported ${ }^{[11]}$. Briefly, the resected omental 
tissue was incubated in the presence of $0.25 \%$ trypsin $/ 0.01 \%$ EDTA at $37^{\circ} \mathrm{C}$ for $20 \mathrm{~min}$. The detached HPMCs were then cultured in RPMI-1640 media (PAA Laboratories GmbH, Austria) supplemented with 20\% FCS, penicillin $(100 \mathrm{U} / \mathrm{mL}) /$ streptomycin $(100 \mu \mathrm{g} / \mathrm{mL}), 0.4 \mathrm{mg} / \mathrm{L}$ hydrocortisone and 5 $\mathrm{mg} / \mathrm{L}$ insulin in fibronectin-coated tissue culture flasks at $37^{\circ} \mathrm{C}$ in a humid atmosphere of $5 \% \mathrm{CO}_{2}$. Cells were used for experiments following 3 rounds of passage.

\section{Recombinant plasmid construction, lentivirus production and transduction}

All the procedures were performed according to the BLOCK- iT ${ }^{\mathrm{TM}}$ Pol II miR RNAi Expression Vector Kit and the Lentiviral Pol II miR RNAi Expression System (Invitrogen, Carlsbad, CA, USA). Four pairs of miR-155-based c-Met targeting sequences (64 bp) and a negative control sequence were designed using the Invitrogen RNAi Designer (www.invitrogen.com/rnaiexpress). The engineered pre-miRNA sequence structure was based on the murine miR-155 sequence, and synthesized double oligonucleotides were cloned into the pcDNA6.2-GW/EmGFP/miR plasmid (Invitrogen) to produce the recombinant plasmids containing either the c-Met miRNA insert (pCMV-c-Met miRNA-825, pCMV-c-Met miRNA-1872, pCMV-c-Met miRNA-3409, and pCMV-c-Met miRNA-4339) or the negative control (pCMV-c-Met miRNA-neg). Each purified expression plasmid was transfected into the SGC7901 cells. RT-PCR and Western blot analysis were performed to assess the efficiency of c-Met knockdown at $48 \mathrm{~h}$ post-transfection.

The lentiviral miRNA system based on the above vectors was generated. The third generation self-inactivating lentivirus vector, pLenti6/V5-DEST containing a CMV-driven EGFP reporter and a SV40 promoter upstream of the cloning sites for high-level expression of miRNA was used. The constructed functional pre-miRNA expression cassette targeting c-Met was transferred into the destination vector (pLenti6/V5-DEST) to generate the lentiviral expression vector (Lenti-c-MetmiRNA) or the negative control vector (Lenti-c-MetmiRNA-neg). The recombinant lentivirus and the control lentivirus were produced by co-transfecting 293FT cells with the transfer vector and the 3 packaging vectors. The virus-containing supernatant was harvested $72 \mathrm{~h}$ post-transfection. The prepared SGC7901 cells were transduced with the lentiviral expression vectors (Lenti-c-MetmiRNA or Lenti-c-MetmiRNA-neg). At $48 \mathrm{~h}$ after transduction, the knockdown of c-Met was evaluated by Western blot analysis. Stable cell lines were obtained after selection by long-term culture in medium containing 4 $\mu \mathrm{g} / \mathrm{mL}$ blasticidin for $12 \mathrm{~d}$.

\section{Proliferation assay}

Cell proliferation was evaluated by 3-(4,5-dimethylthiazol2-yl)-2,5-diphenyltetrazolium bromide (MTT, Sigma, USA) assay. Cells were seeded at a density of $5 \times 10^{3}$ in 96 -well plates in Dulbecco's modified Eagle's medium (DMEM) containing $10 \%$ FCS in a final volume of $100 \mu \mathrm{L}$. After incubation for $18 \mathrm{~h}$, the cells grew to approximately $85 \%$ confluency, and then were transduced with the lentivirus (50 $\mu \mathrm{L} /$ well). At 24, 48, 60 , and $96 \mathrm{~h}$ post-transduction, an MTT assay was performed. Cells were incubated in the presence of MTT $(20 \mu \mathrm{L} /$ well of $5 \mathrm{~g} / \mathrm{L}$ solution in sterile PBS) for $4 \mathrm{~h}$ at $37^{\circ} \mathrm{C}$. The assay was read by determination of the reaction product at $490 \mathrm{~nm}$ by using a microplate reader (Multiskan MK3, Thermo Labsystems, Finland).

\section{Invasive assay}

The ability of cells to invade through ECM was assessed using the Chemicon QCM 96-well Invasion Assay Kit (ECM555, CHEMICON, International, CA, USA). Cells $\left(3 \times 10^{6}\right)$ were harvested with trypsin/EDTA, and suspended in PRMI-1640 media with $0.5 \%$ FBS. The invasive assay was performed according to the manufacturer's instructions. Briefly, the invasive gastric cancer cells, which migrated through the ECM layer and attached to the bottom of the polycarbonate membrane, were dissociated from the membrane after incubation with the Cell Detachment Solution for $30 \mathrm{~min}$ at $37^{\circ} \mathrm{C}$. Next, $50 \mu \mathrm{L}$ of lysis buffer/CyQuant GR Dye Solution (1:75) was added to each well and incubated for $15 \mathrm{~min}$ at room temperature. Finally, $150 \mu \mathrm{L}$ of the mixture was transferred to a new 96-well plate, and the fluorescence value was detected with a fluorescence plate reader using $480 \mathrm{~nm} / 520 \mathrm{~nm}$ filter set. As a negative control, Cell Detachment Solution in the absence of SGC7901 cells was used.

\section{Adhesion of gastric cancer cells to a HPMCs monolayer in vitro}

Tumor cells were fluorescently labeled with $5 \mu \mathrm{mol} / \mathrm{mL}$ BCECF/AM (Biotium, Hayward, CA, USA). HPMCs $\left(1.5 \times 10^{4}\right.$ cells/well) were cultured in 96-well plates, which were precoated with $0.1 \%$ gelatin, until confluent. SGC7901 cells $\left(3 \times 10^{4}\right)$ were resuspended in $200 \mu \mathrm{L}$ medium and added to each well. The plates were incubated for $60 \mathrm{~min}$ at $37^{\circ} \mathrm{C}$. The number of adhesive cells was quantified by measuring the fluorescence intensity on a Perkin Elmer plate reader (CA, USA) with $488 \mathrm{~nm}$ excitation and $535 \mathrm{~nm}$ emission filters.

\section{Adhesion of gastric cancer cells to mice peritoneum in vivo}

Six-week-old BALB/c athymic female nude mice (Shanghai Laboratory Animal Center, Shanghai, China) were used in this study. The mice were divided into three groups (10 mice per group): SGC7901, Lenti-miRNAc-Met and Lenti-miRNAcMet-neg. Cultured tumor cells, fluorescently labeled as above, were injected at a concentration of $2 \times 10^{6} / \mathrm{mL}$ into the mice peritoneal cavity in a final volume of $1 \mathrm{~mL}$. After $4 \mathrm{~h}$, mice were sacrificed, and the full peritoneum was cut into $1 \mathrm{~cm}^{2}$ pieces and placed in a 24-well culture plate in DMEM containing $10 \%$ FCS. Adherent cells were lysed with 1\% NP-40, and the fluorescence intensity was measured. All animal experiments were conducted in accordance with the Guidelines for the Care and Use of Laboratory Animals of Tongji University.

\section{Reverse transcription-PCR (RT-PCR)}

Total RNA was extracted with TRIzol Reagent (Invitrogen). PCR was performed using the RT-PCR Kit (Toyobo, Japan) 
with $5 \mu \mathrm{g}$ total RNA to synthesize cDNA. The primers of c-Met were 5'-CCTGCGAAGTGAAGGGTCTCC-3' (forward primer) and 5'-CTGGCAGCTTTGCACCTGTTT-3' (reverse primer). GAPDH was used as an internal control. The primers of human GAPDH were 5'-ACCACAGTCCATGCCATCAC-3' (forward primer) and 5'-TCCACCACCCTGTTGCTGTA-3' (reverse primer). PCR was performed in $30 \mu \mathrm{L}$ of reaction mixture. Thermal cycle conditions were conducted with preamplification denaturation at $95^{\circ} \mathrm{C}$ for $3 \mathrm{~min}$, followed by 30 cycles of denaturation at $95^{\circ} \mathrm{C}$ for $20 \mathrm{~s}$, annealing at $62^{\circ} \mathrm{C}$ for $20 \mathrm{~s}$, and extension at $72^{\circ} \mathrm{C}$ for $30 \mathrm{~s}$, with a final extension at $72{ }^{\circ} \mathrm{C}$ for $3 \mathrm{~min}$. For the semi-quantification, an image of the gel was captured, and the intensity of the bands was quantified by gel analysis system (Bio-Rad, USA).

\section{Western blot analysis}

SGC7901 cells $\left(5 \times 10^{6}\right)$ were collected and lysed in cold PBS with $0.5 \%$ NP-40 and a protease inhibitor cocktail (Novogen, Australia). Whole-cell proteins were extracted using the Whole Cell Extraction Kit (Chemicon). Protein concentrations were determined according to the manufacturer's instructions of the BCA (bicinchoninic acid) Protein Assay Kit (Pierce, Rockford, USA). Samples were equalized, and $40 \mathrm{mg}$ of total protein for each sample was loaded into separate lanes on a $12 \%$ SDS-PAGE and transferred onto a nitrocellulose membrane (Invitrogen). Western blot analysis was performed using primary antibody for c-Met (1:100 dilution, Santa Cruz Biotechnology, CA, USA). Protein expression was quantified by densitometry and normalized to $\beta$-actin (1:100, Santa Cruz) expression using enhanced chemiluminescence (Pierce, Rockford, IL, USA).

\section{Adhesion molecule expression as detected by flow cytometry} (FCM)

Tumor cells $\left(3 \times 10^{6}\right)$ were incubated with primary antibodies (Integrin $\beta 11.5 \mu \mathrm{g}$, E-cadherin $1.5 \mu \mathrm{g}$, CD44 $0.75 \mu \mathrm{g}$, Biolegend, San Diego, CA, USA) for $30 \mathrm{~min}$ at $4^{\circ} \mathrm{C}$. PE-labeled secondary antibody was added and incubated for $30 \mathrm{~min}$. Additionally, the primary antibody PE-a3-integrin (BioLegend, San Diego, CA, USA) was incubated with tumor cells for $30 \mathrm{~min}$. Next, cells were resuspended in $500 \mu \mathrm{L}$ of $1 \%$ BSA/PBS. Fluorescence expressions on cells surface were quantitatively analyzed with a FACSCalibur (Becton-Dickinson, San Jose, USA). Cells incubated in the absence of primary antibody were set as a blank, and those incubated in the isotype-specific hamster IgG1-PE were set as a negative control to eliminate nonspecific staining.

\section{In vivo studies of peritoneal dissemination model in nude mice}

BALB/c female nude mice (5-6 weeks of age) were purchased from the Shanghai Laboratory Animal Center (Shanghai, China). Thirty mice were allocated to three groups (10 mice per group): SGC7901 (group A), Lenti-miRNAc-Met-neg (group B) and Lenti-miRNAc-Met (group C). Suspensions of tumor cells $\left(1 \times 10^{7}\right.$ cells $)$ in $1 \mathrm{~mL}$ RPMI-1640 media were injected into the peritoneal cavity of the mice for incubation.
On d 30, all thirty mice were sacrificed.

To evaluate further the treatment effect of Lenti-miRNAcMet, another 45 nude mice were allocated to three groups as above (15 mice per group). In the PBS group, $4 \mathrm{~mL}$ PBS was injected into the peritoneal cavity of each mouse. In the Lenti-miRNAc-Met-neg group, $5 \times 10^{7}$ copies $/ 4 \mathrm{~mL}$ of LentimiRNAc-Met-neg was injected in each mouse intraperitoneally (ip). In the Lenti-miRNAc-Met group, $5 \times 10^{7}$ copies $/ 4 \mathrm{~mL}$ of Lenti-miRNAc-Met was injected in each mouse ip. Three days postinoculation, $1 \times 10^{7}$ SGC7901 cells in $1 \mathrm{~mL}$ PBS were injected into each mouse ip. On d 30, 10 mice from each group were sacrificed. The remaining five mice in each group were used to evaluate the survival up to $\mathrm{d} 120$. The macroscopic nodules on peritoneal surface were counted, and tumor size of large nodules that exceeded $1.0 \mathrm{~cm}$ in diameter was calculated by assuming a spherical shape. The fused nodules were counted as a single nodule. All animal experiments were conducted in accordance with the Guidelines for the Care and Use of Laboratory Animals of Tongji University.

\section{Statistical analysis}

Statistical analysis was performed using the Statistics Package for the Social Science software (version 11.5; SPSS Inc, Chicago, IL, USA). The comparison between different groups was analyzed by the Independent Samples $t$ Test or the Mann-Whitney Test. Survival curves were obtained using the Kaplan-Meier method and compared by the log-rank test. All the statistical analysis was two sides with significance defined as $P<0.05$.

\section{Results}

\section{Expression of c-Met in the PFCCs}

PFCCs were found utilizing MACS in the ascites of 6 out of 10 gastric cancer patients. PFCCs had an increased nucleus: cytoplasm ratio with nuclear hyperchromasia observed by light microscope (Figure 1Aa). CK20-positive expression verified their epithelial origin, notably from gastrointestinal tract (Figure 1Ab). c-Met expression was detected in the PFCCs of 5 patients (Figure $1 \mathrm{Ac}$ ).

\section{Lentivirus-mediated RNAi significantly inhibited c-Met expression in SGC7901 cells}

Four recombinant expression plasmids targeting c-Met were constructed. The mRNA and protein expression levels of c-Met in SGC7901 cells were examined by RT-PCR and Western blot analysis at $48 \mathrm{~h}$ post-transfection. As shown in Figure $1 \mathrm{~B}$ and $1 \mathrm{C}$, when compared with the parental SGC7901 cells, the negative control did not affect the expression levels of c-Met, whereas pCMV-c-MetmiRNA-825 resulted in the decrease of both c-Met mRNA and protein levels. Specifically, c-Met mRNA expression was reduced by $80 \%-83 \%$, while c-Met protein expression was reduced by $88 \%-90 \%$ in pCMVc-MetmiRNA-825 transfected cells.

The recombinant expression plasmid pCMV-c-MetmiRNA-825 was inserted into pLenti6/V5-DEST destination vector to develop a Lenti-miRNA expression vector. We infected SGC7901 cells with the lentivirus carrying pCMV- 
A

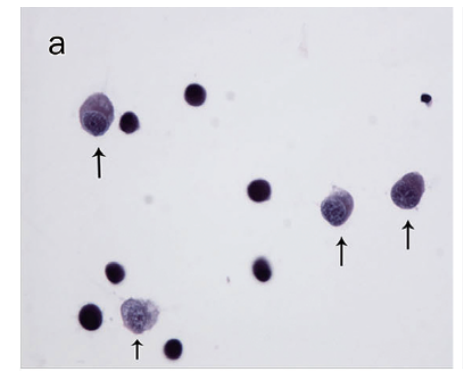

b

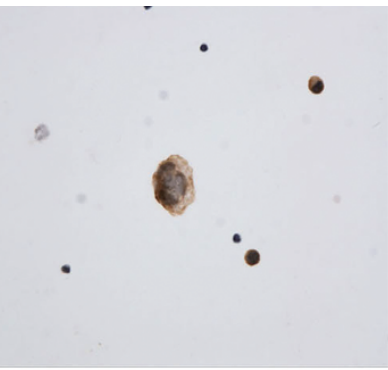

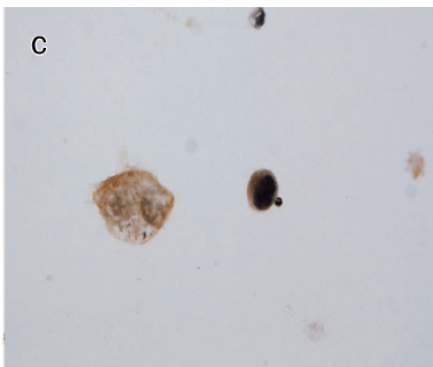

B a

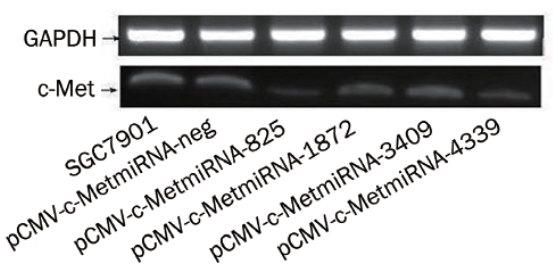

b

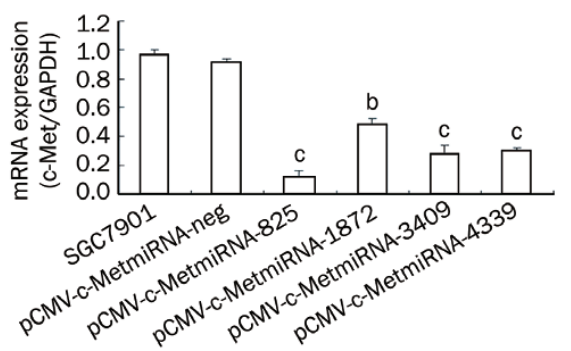

C a

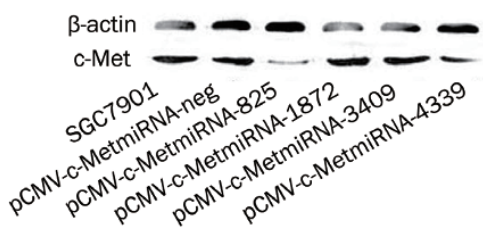

b

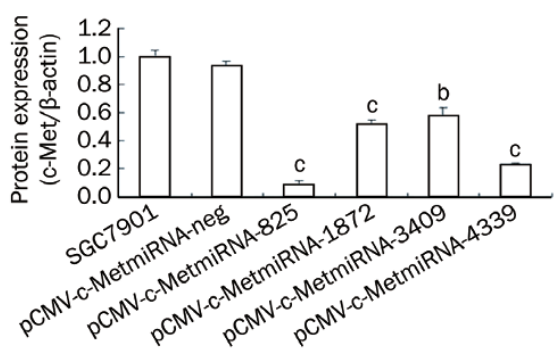

Figure 1. Expression of C-Met in the PFCCs and SGC7901 cells. (A) Free malignant cells detected by MACS from the ascites of gastric cancer patients. (a) HE staining (arrows, magnification 200×). (b) CK20 expression in the free gastric cancer cells (immunocytochemical staining, magnification 200x). (c) c-Met expression in the free gastric cancer cells (immunocytochemical staining, magnification 200×). (B) Expression of c-Met mRNA in SGC7901 cells transduced with pCMV-c-MetmiRNA expression plasmids at $48 \mathrm{~h}$. (a) RT-PCR assay. (b) c-Met mRNA in pCMV-c-MetmiRNA-825 transduced cells was significantly reduced by $87.6 \%$ when compared with parental SGC7901 cells $\left({ }^{b} P<0.05,{ }^{c} P<0.01\right)$. (C) Expression of c-Met protein in SGC7901 cells transduced with different pCMV-c-MetmiRNA expression plasmids at $48 \mathrm{~h}$. (a) Western blot assay. (b) c-Met protein in pCMV-c-MetmiRNA-825 transduced cells was significantly reduced by $90 \%$ when compared with the parental SGC7901 cells $\left({ }^{b} P<0.05,{ }^{c} P<0.01\right)$.

c-MetmiRNA-825 or pCMV-c-MetmiRNA-neg. c-Met protein expression decreased by $91.5 \%$ in Lenti-miRNAc-Met transfected cells as compared with the parental SGC7901 cells $(P<0.05$, Figure 2A, 2B) $48 \mathrm{~h}$ post-transfection. A stable SGC7901 cell line transduced with lentivirus was successfully generated by blasticidin $(4 \mathrm{\mu g} / \mathrm{mL})$ selection. After continuous culture for $30 \mathrm{~d}$, the lentivirus-transduced cells had a stable inhibition of c-Met expression (EGFP positive expression in cells was $78.6 \%$, Figure 2C).

\section{Knockdown of c-Met suppressed cell proliferation in vitro}

The effect of knockdown of c-Met expression via LentimiRNAc-Met on gastric cancer cell proliferation was determined by MTT assay. As shown in Figure 3, cell proliferation was inhibited in a time-dependent manner in SGC7901 cells transfected with Lenti-miRNAc-Met for 24-96 h post-transduction when compared with the parental cells and negative- control groups.

\section{Knockdown of c-Met suppressed cell invasion in vitro}

To verify the effect of knockdown of c-Met expression via Lenti-miRNAc-Met on migration ability of SGC7901 cells, an invasion assay was performed. Migration was determined in accordance with the fluorescence value, which represented the invading SGC7901 cells that migrated through the ECM layer. Figure 4 demonstrated that the fluorescence value of the cells transduced with Lenti-miRNAc-Met (23191.21 \pm 5183.91$)$ was much lower than the parental cells $(45496.13 \pm 9408.99)$ or the negative control cells (42821.36 \pm 9513.45$)$, indicating the invasive capacity of the Lenti-miRNAc-Met transduced cells markedly decreased. This result suggested that Lenti-miRNAc-Met attenuated the metastatic potential of gastric cancer cells in vitro. 
A

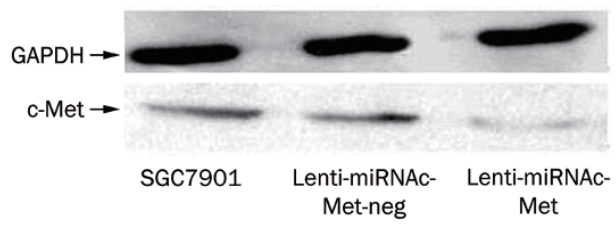

B

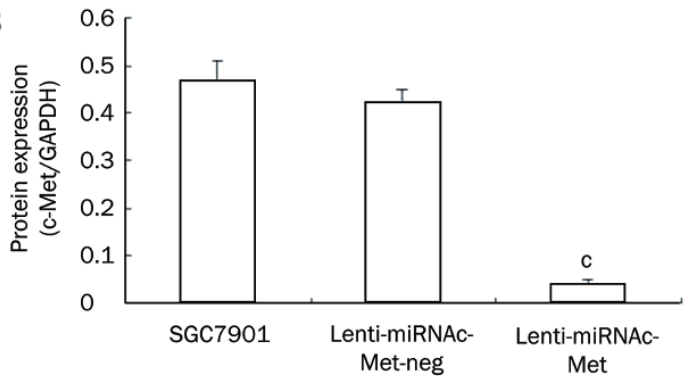

C

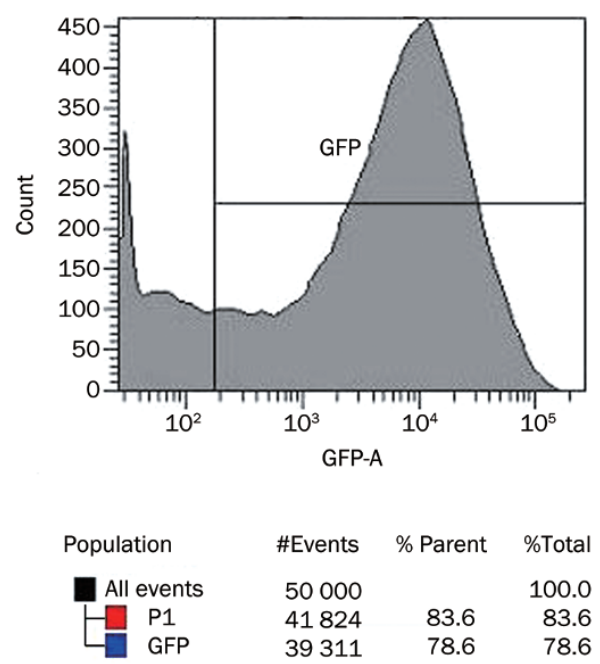

Figure 2. Lentivirus-mediated RNAi suppressed c-Met protein expression in SGC7901 cells. (A) Western blot assay. (B) c-Met protein in Lenti-miRNAcMet transduced cells was significantly reduced by $91.5 \%$ when compared with the parental SGC7901 cells $\left({ }^{\circ} P<0.01\right)$. (C) The transduction efficiency after continuous culture in the lentivirus-miRNAc-Met stably transduced cell line was $78.6 \%$ as measured by FCM.

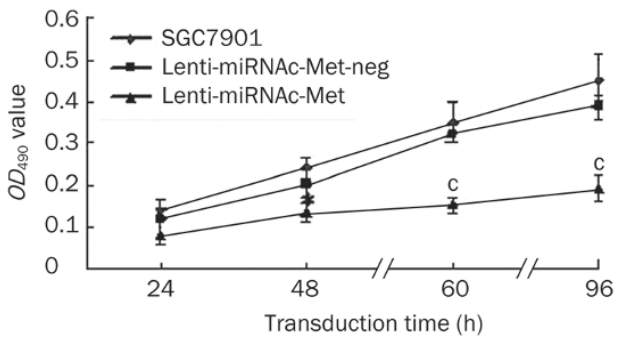

Figure 3. Cell growth was significantly inhibited in SGC7901 cells after transduction with Lenti-miRNAc-Met at various times as measured by MTT assay $\left({ }^{\circ} P<0.01\right)$.
Knockdown of c-Met suppressed cell adhesion to HPMCs in vitro and mouse peritoneum in vivo by downregulation of integrin $\alpha 3 \beta 1$ and E-cadherin

Cancer cell adhesion to peritoneum is a crucial process and the initial step during peritoneal metastasis. To determine the effect of knockdown of c-Met expression via Lenti-miRNAcMet on adhesion, fluorescently labeled SGC7901 cells were tested via an adhesion assay. In vitro, the adhesion of the Lenti-miRNAc-Met transduced SGC7901 cells to the monolayer of HPMCs was decreased by $64 \%$ (Figure 5A1, $P<0.01$ ), and in vivo, adhesion to the full-thickness mice peritoneum decreased by $69 \%$ (Figure 5A2, $P<0.01$ ), both compared with
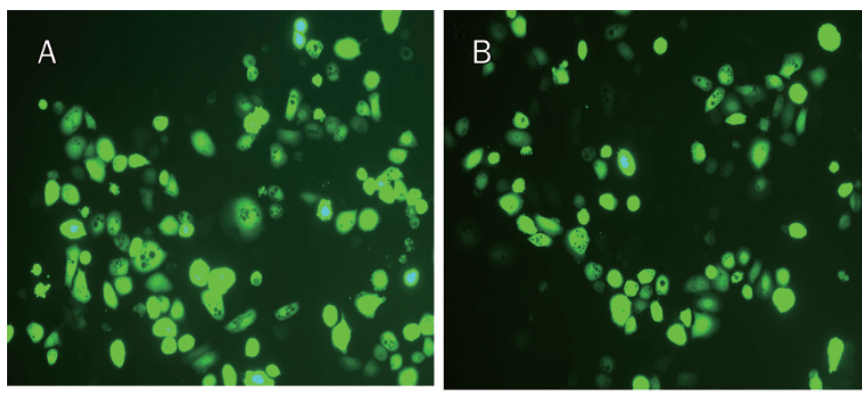

D

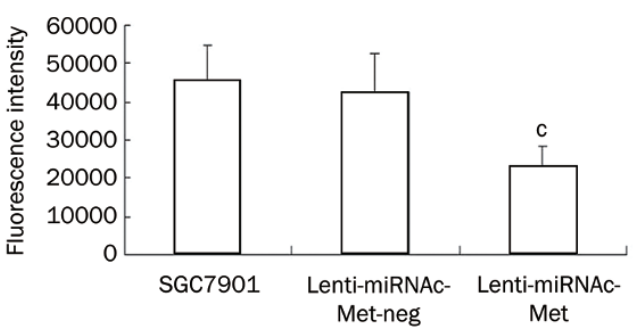

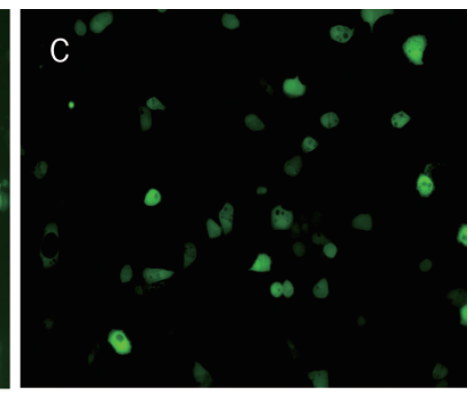

Figure 4. The ability of SGC7901 cells transduced with Lenti-miRNAc-Met to invade through ECM as detected by an invasion assay. (A) SGC7901 cells. (B) Lenti-miRNAc-Met-neg transduced cells. (C) Lenti-miRNAc-Met transduced cells. (D) The invasion to ECM was significantly inhibited in the cells transduced with Lenti-miRNAc-Met when compared with the parental SGC7901 cells according to the fluorescence assay $\left({ }^{\circ} P<0.01\right)$. 
A1
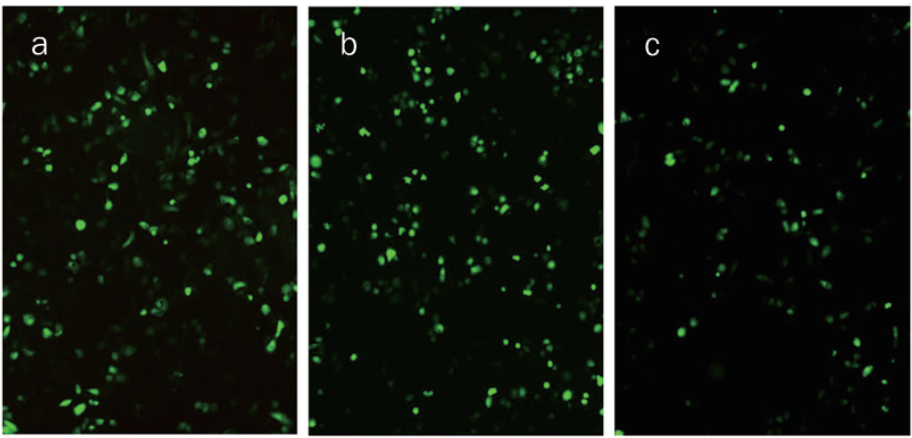

A2
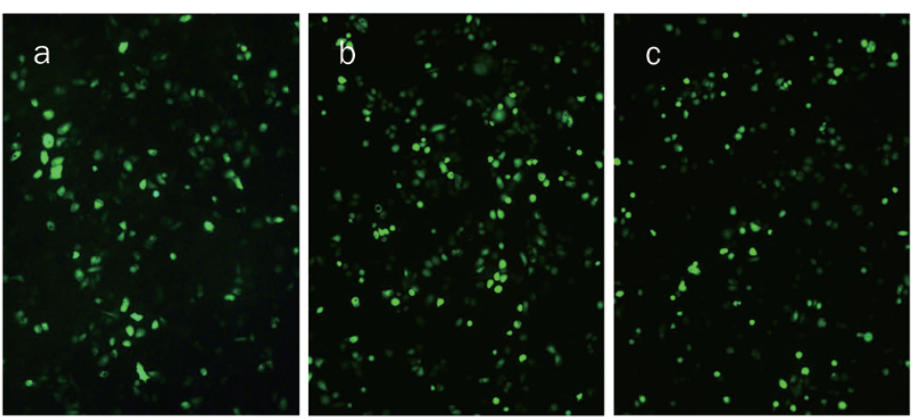

d

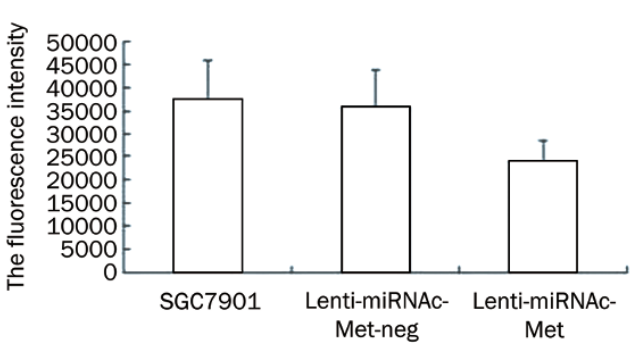

d

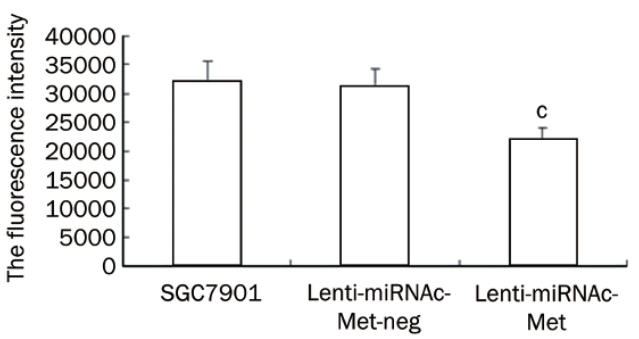

B

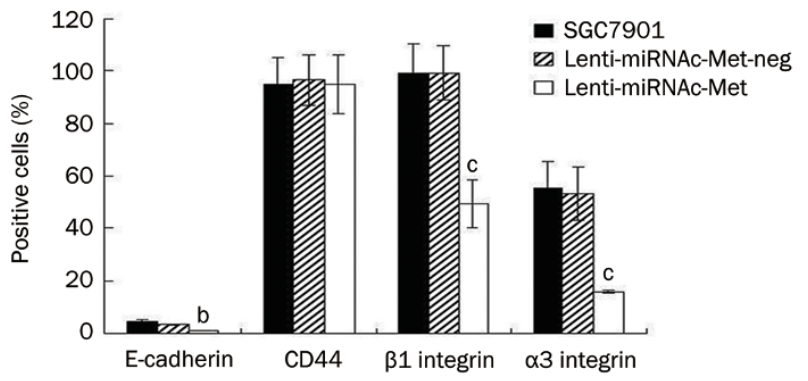

the parental cells. No statistical significance was observed between the SCG7901 cells and the negative control $(P>0.05)$.

To investigate the mechanism of adhesion to the peritoneum, adhesion molecule expression was examined by FCM. As shown in Figure 5B, the expressions of E-cadherin, integrin $\beta 1$ and integrin a3 were significantly reduced in LentimiRNAc-Met transduced SGC7901 cells after 48 h, compared with the SGC7901 cells (Figure 5B, P<0.01). However, CD44 expression was not significantly inhibited (Figure $5 B, P>0.05$ ).

\section{Knockdown of c-Met inhibited tumorigenicity of SGC7901 cells in} vivo

To observe the inhibition of peritoneal dissemination of SGC7901 cells in vivo after knockdown of c-Met, we inoculated nude mice ip with $1 \times 10^{7}$ parental, Lenti-miRNAc-Met-neg transduced or Lenti-miRNAc-Met transduced SGC7901 cells. At $30 \mathrm{~d}$ postinoculation, numerous tumor nodules of peritoneal dissemination were observed on the peritoneal surface around the radix of mesentery and blood vessels in SGC7901 group and the negative control group, whereas tumor nodules were significantly suppressed in mice in the Lenti-miRNAcMet inoculated group $(P<0.05$, Figure 6$)$. The size of large
Figure 5. Adhesion of SGC7901 cells transduced with Lenti-miRNACMet detected by the fluorescence value assay $(A)$ and their expressions of adhesion molecules detected by FCM (B) $\left({ }^{b} P<0.05,{ }^{c} P<0.01\right)$. (A1) Adhesion of SGC7901 cells transduced with Lenti-miRNAc-Met to HPMCs monolayer in vitro was significantly inhibited when compared with parental SGC7901 cells. (A2) Adhesion of SGC7901 cells transduced with LentimiRNAc-Met to mice peritoneum in vivo was significantly inhibited when compared with parental SGC7901 cells. (B) The adhesion molecules expressions in SGC7901 cells. Groups: (a) SGC7901 cells; (b) LentimiRNAc-Met-neg transduced cells; (c) Lenti-miRNAc-Met transduced cells.

nodules that exceeded $1.0 \mathrm{~cm}$ in diameter was also significantly reduced compared with SGC7901 and the negative control inoculated animals $(P<0.05$, Figure 6A3).

\section{Treatment of peritoneal dissemination by Lenti-miRNAc-Met in} nude mice

To further study the efficacy of Lenti-miRNAc-Met as a therapeutic target for peritoneal dissemination in gastric cancer, three days after we injected Lenti-miRNAc-Met and the control lentivirus, we established a peritoneal dissemination model by intraperitoneal incubation with $1 \times 10^{7}$ SGC7901 cells in nude mice. Peritoneal dissemination was significantly inhibited in the group treated with Lenti-miRNAc-Met as compared with the control group treated with Lenti-miRNAcMet-neg or PBS $(P<0.05$, Figure 6B). The mice treated with Lenti-miRNAc-Met-neg or PBS died within $75 \mathrm{~d}$. However, the mice treated with Lenti-miRNAc-Met had prolonged lives up to $116 \mathrm{~d}(P<0.05$, Figure 6B2).

\section{Discussion}

The biological function of PFCCs can be considered a decisive factor in tumor peritoneal dissemination and the basis for ther- 
A1
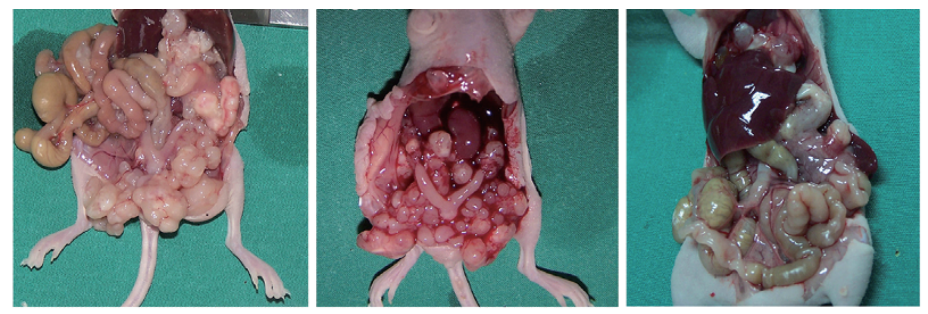

A2

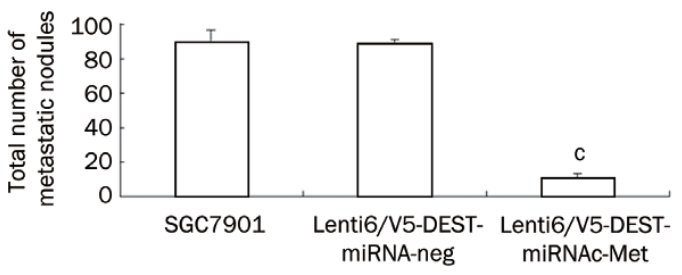

A3

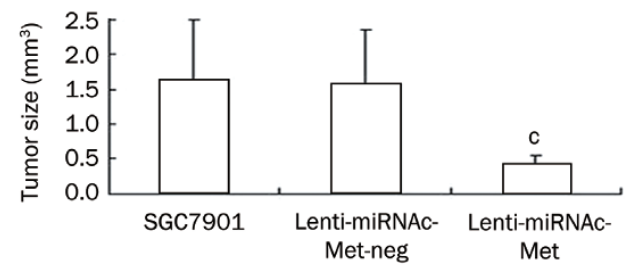

B1
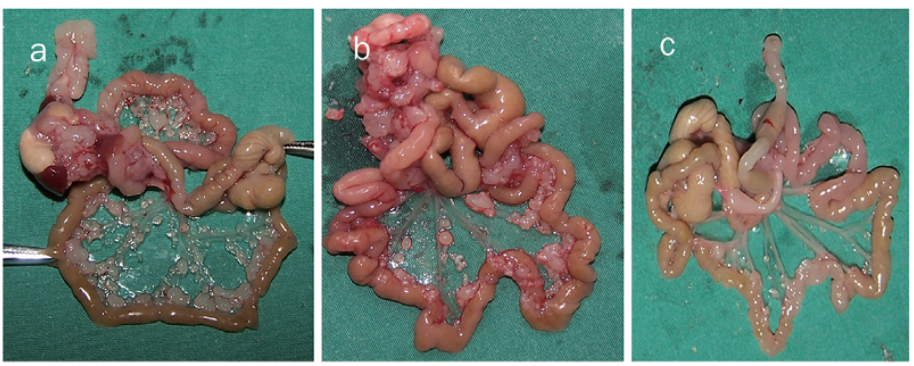

B2

- Lenti-miRNA-c-Met

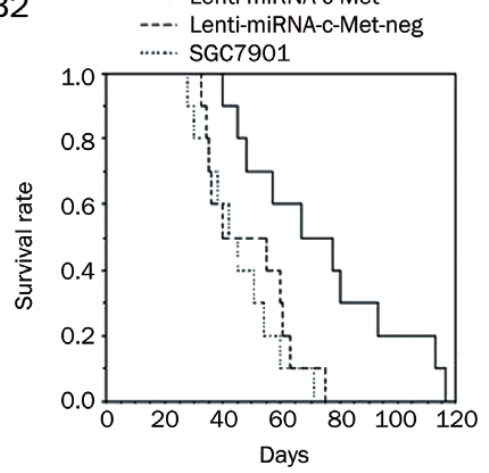

B3

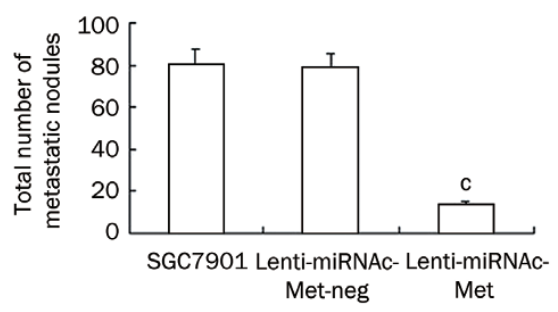

B4

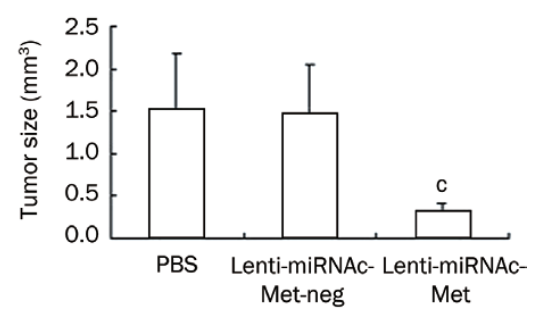

Figure 6. Lentivirus-mediated RNAi silencing of c-Met effectively suppresses peritoneal dissemination of gastric cancer in vitro and in vivo. Groups: (a) SGC7901; (b) Lenti-miRNAc-Met-neg; (c) Lenti-miRNAc-Met. (A) Tumor growth in the abdominal cavities of mice was inhibited by suppression of c-Met using Lenti-miRNAc-Met. (A1) Tumor nodules on peritoneal surface and around the radix of mesentery. (A2) The total number of tumor nodules was significantly reduced in Lenti-miRNAc-Met transduced cells when compared with the parental SGC7901 cells ( ${ }^{\circ} P<0.01$ ). (A3) The tumor size was significantly reduced in Lenti-miRNAc-Met transduced cells when compared with the parental SGC7901 cells $\left({ }^{\circ} P<0.01\right)$. (B) Intraperitoneal injection of Lenti-miRNAc-Met significantly suppressed tumor growth in a nude mouse peritoneal dissemination model of gastric cancer. (B1) Tumor nodules on peritoneal surface and around the radix of mesentery were significantly reduced in the Lenti-miRNAc-Met group (c), when compared with the PBS group (a) and the Lenti-miRNAc-Met-neg group (b). (B2) The survival rate of the mice treated with Lenti-miRNAc-Met was higher than those of the mice treated with Lenti-miRNAc-Met-neg or PBS. (B3) The total number of tumor metastatic nodules was significantly reduced in mice treated with Lenti-miRNAcMet when compared with Lenti-miRNAc-Met-neg or PBS ( $\left.{ }^{\circ} P<0.01\right)$. (B4) The tumor size was significantly reduced in mice treated with Lenti-miRNAc-Met when compared with Lenti-miRNAc-Met-neg or PBS $\left({ }^{\circ} P<0.01\right)$.

apeutic strategy. Isolation of PFCCs is helpful to understand their biological characteristics. However, early detection of rare free cancer cells in the abdominal cavity is difficult with conventional methods. In this study, we successfully isolated
PFCCs using the MACS method in the ascites from 6 out of 10 gastric cancer patients, and moreover, c-Met expression was detected in the PFCCs of 5 patients.

The general indication for treatment with kinase and sig- 
naling pathway inhibitors is based on the level of expression and mutation status in cancer cells. For example, the effect of targeting human epidermal growth factor receptor-2 (HER-2) therapy in breast cancer depends on the level of HER-2 expression in breast cancer cells. Gastric cancer as a common cancer leading to peritoneal dissemination, the peritoneum is a common site for distant metastasis. Though the previous study has shown the effect of targeting c-Met therapy in ovarian cancer in vivo ${ }^{[11]}$, the potential therapeutic efficacy of c-Met for gastric cancer has not been studied. Therefore, the expression of c-Met in PFCCs from gastric cancer ascites suggests that it may have the possibility to develop a treatment targeting c-Met for peritoneal dissemination in gastric cancer patients.

Due to that peritoneal dissemination is characterized by the adhesion and invasion of tumor cells into the peritoneum ${ }^{[11]}$, it is an important process that cancer cells detaching from the primary cancer and successfully adhering to mesothelial cells in the dissemination and formation of metastases in gastric cancer. In this study, using lentivirus mediated RNAi technique, the expression of c-Met in both mRNA (80\%-83\%) and protein levels ( $88 \%-90 \%)$ were effectively knocked down by artificial engineered pre-miRNA sequences specifically targeting c-Met in human SGC7901 gastric cancer cell line. Moreover, we established the stably transduced Lenti-miRNAcMet SGC7901 cell line and the control stably transduced cell line. After knockdown of c-Met expression, the adhesion of SGC7901 gastric cancer cells to monolayer HPMC in vitro and to mice peritoneum in vivo were significantly suppressed, compared with the control virus.

Many adhesion molecules are involved in this regulation as the previous reported. We further investigated the mechanism of adhesion. The findings showed that E-cadherin and a3 $\beta 1$ integrin expression were reduced in the Lenti-miRNAcMet transduced SGC7901 cells, as compared with the negative control and parental cells. However, no close relationship between c-Met and CD44 in our study was found although some CD44 isoforms can promote or increase c-Met activation $^{[16]}$. E-cadherin is also involved in the multistep process of peritoneal dissemination in epithelial ovarian carcinoma ${ }^{[2]}$. c-Met can remove E-cadherin from cell-cell adhesion sites and regulate the activity of integrins ${ }^{[17]}$. In gastric cancer, modulation of E-cadherin by hepatocyte growth factor/c-Met induces aggressiveness of gastric carcinoma ${ }^{[18]}$. CD44 can cause very strong cell adhesion to peritoneal mesothelium and an unfavourable prognosis in ovarian cancer ${ }^{[3]}$, but we did not find the similar result in gastric cancer in this study. a3 $\beta 1$ integrin plays an essential role in mediating the initial attachment of cancer cells to the peritoneum in the peritoneal implantation of NUCC-4 human gastric cancer cells in athymic mice ${ }^{[8]}$. Therefore, c-Met can regulate these molecules in the peritoneal dissemination of gastric cancer. However, in ovarian cancer, targeting c-Met in vivo inhibited peritoneal dissemination and invasion through an a5 $\beta 1$ integrin-dependent mechanism ${ }^{[11]}$. So, in human cancers, various integrin combinations of a and $\beta$ subunits produce polymorphisms with different ligands specificity and function. The molecules involved in peritoneal dissemination may also be different.

After adhesion to peritoneum, cancer cells invade the ECM of the peritoneum and form the metastasis site. c-Met plays an important role in the process of epithelial-mesenchymal interaction, regulation of cell migration, invasion, cell proliferation and survival. Our results indicated the Lenti-miRNAc-Met transduced SGC7901 cells survival and their ability to invade the ECM were significantly inhibited in vitro.

The biological function of HGF-MET axis affects tumor growth and development of metastasis ${ }^{[19]}$. The advantage of stable and long-term gene silencing offered by the lentivirus vector system makes it a powerful tool for gene therapy. Based on the peritoneal metastasis model established by SGC7901 gastric cancer cells ${ }^{[20]}$, we found that tumor growth (including the number of macroscopic nodules on peritoneal surface and tumor size of large nodules) in mice which received intraperitoneal injections of SGC7901 cells transduced with Lenti-miRNAc-Met was strongly suppressed compared with the Lenti-miRNAc-Met-neg control and parental SGC7901 cell groups. The intraperitoneal administration of Lenti-miRNAc-Met dramatically suppressed the growth of gastric cancer cells on the surface of peritoneum and improved the survival of the mice. Combining these findings in vitro and in vivo, we confirmed the role of c-Met in the growth, proliferation, invasion to ECM, adhesion to peritoneum and peritoneal dissemination in gastric cancer.

In summary, our findings indicate that c-Met is expressed in PFCCs, and it plays an important role in the process of gastric cancer peritoneal dissemination, including tumor growth, adhesion to peritoneum and invasion to ECM. E-cadherin and $a 3 \beta 1$ integrin are involved in the regulation of adhesion of SGC7901 cells to mesothelial cells. Therefore, Lentivirusmediated RNAi targeting c-Met may be a useful tool for gene therapy targeting gastric cancer peritoneal dissemination.

\section{Acknowledgements}

This work was supported in part by the Natural Science Foundation of Shanghai (№ 05ZR14130).

\section{Author contribution}

Xiao-lei WANG was the guarantor of integrity of the entire study, responsible for study design, data analysis and manuscript preparation. Xi-mei CHEN provided final approval of the version to be submitted. Jian-ping FANG participated in the immunocytochemical staining experiments. Chang-qin YANG revised the article for important intellectual content.

\section{References}

1 Yonemura Y, Endo Y, Obata T, Sasaki T. Recent advances in the treatment of peritoneal dissemination of gastrointestinal cancers by nucleoside antimetabolites. Cancer Sci 2007; 98: 11-8.

2 Terauchi M, Kajiyama H, Yamashita M, Kato M, Tsukamoto H, Umezu T, et al. Possible involvement of TWIST in enhanced peritoneal metastasis of epithelial ovarian carcinoma. Clin Exp Metastasis 2007; 24: 329-39.

3 Li CZ, Liu B, Wen ZQ, Li HY. Inhibition of CD44 expression by small 
interfering RNA to suppress the growth and metastasis of ovarian cancer cells in vitro and in vivo. Folia Biol (Praha) 2008; 54: 180-6.

4 Hynes RO. Integrins: bidirectional, allosteric signaling machines. Cell 2002; 110: 673-87.

5 Jin H, Varner J. Integrins: roles in cancer development and as treatment targets. Br J Cancer 2004; 90: 561-5.

6 Knudsen BS, Vande Woude G. Showering c-MET-dependent cancers with drugs. Curr Opin Genet Dev 2008; 18: 87-96.

7 Ayhan A, Ertunc D, Tok EC, Ayhan A. Expression of the c-Met in advanced epithelial ovarian cancer and its prognostic significance. Int J Gynecol Cancer 2005; 15: 618-23.

8 Drebber U, Baldus SE, Nolden B, Grass G, Bollschweiler E, Dienes HP, et al. The overexpression of c-met as a prognostic indicator for gastric carcinoma compared to p53 and p21 nuclear accumulation. Oncol Rep 2008; 19: 1477-83.

9 Tuynman JB, Lagarde SM, Ten Kate FJ, Richel DJ, van Lanschot JJ. Met expression is an independent prognostic risk factor in patients with oesophageal adenocarcinoma. Br J Cancer 2008; 98: 1102-8.

10 Zeng ZS, Weiser MR, Kuntz E, Chen CT, Khan SA, Forslund A, et al. c-Met gene amplification is associated with advanced stage colorectal cancer and liver metastases. Cancer Lett 2008; 265: 258-69.

11 Sawada K, Radjabi AR, Shinomiya N, Kistner E, Kenny H, Becker AR, et al. c-Met overexpression is a prognostic factor in ovarian cancer and an effective target for inhibition of peritoneal dissemination and invasion. Cancer Res 2007; 67: 1670-9.

12 Yonemura Y, Endo Y, Obata T, Sasaki T. Recent advances in the treatment of peritoneal dissemination of gastrointestinal cancers by nucleoside antimetabolites. Cancer Sci 2007; 98: 11-8.

13 Barker SD, Casado E, Gomez-Navarro J, Xiang J, Arafat W, Mahasreshti $P$, et al. An immunomagnetic-based method for the purification of ovarian cancer cells from patient-derived ascites. Gynecol Oncol 2001; 82: 57-63.

14 Clement JH, Schwalbe M, Buske N, Wagner K, Schnabelrauch M, Görnert $\mathrm{P}$, et al. Differential interaction of magnetic nanoparticles with tumor cells and peripheral blood cells. J Cancer Res Clin Oncol 2006; 132: 287-92.

15 Ross JS, Carlson JA, Brock G. miRNA: The new gene silencer. Am J Clin Pathol 2007; 128: 830-6.

16 Orian-Rousseau V, Chen L, Sleeman JP, Herrlich P, Ponta H. CD44 is required for two consecutive steps in HGF/c-Met signaling. Genes Dev 2002; 16: 3074-86.

17 Garmy-Susini B, Varner JA. Roles of integrins in tumor angiogenesis and lymphangiogenesis. Lymphat Res Biol 2008; 6: 155-63.

18 Han SU, Lee HY, Lee JH, Kim WH, Nam H, Kim H, et al. Modulation of $\mathrm{E}$-cadherin by hepatocyte growth factor induces aggressiveness of gastric carcinoma. Ann Surg 2005; 242: 676-83.

19 Lesko E, Majka M. The biological role of HGF-MET axis in tumor growth and development of metastasis. Front Biosci 2008; 13: 1271-80.

20 Li Z, Zhan W, Wang Z, Zhu B, He Y, Peng J, et al. Inhibition of PRL-3 gene expression in gastric cancer cell line SGC7901 via microRNA suppressed reduces peritoneal metastasis. Biochem Biophys Res Commun 2006; 348: 229-37. 